\title{
Protein structure reports in JIMD - an important enhancement of journal scope
}

\author{
Bernhard Rupp
}

Published online: 3 March 2011

(C) SSIEM and Springer 2011

"Almost all aspects of life are engineered at the molecular level, and without understanding molecules we can only have a very sketchy understanding of life itself."

Francis Crick, in What Mad Pursuit: A Personal View of Scientific Discovery (1988), p61.

Since the opening quote cited above was stated by Nobel Laureate Francis Crick, more than 20 years of rapid developments in macromolecular crystallography have led to an unprecedented increase of protein structure models publicly available in the worldwide Protein Data Bank (Berman et al. 2003) - the wwPDB contains over 60,000 structure coordinate entries as of this date. While the relevance of structure-function relationships for the understanding and treatment of metabolic diseases is well established, it is generally much harder to actually find and adequately analyze relevant structures. Given the fierce competition for limited space in the high impact 'magazine' journals, many structures of potential interest for the metabolic disease community end up either unpublished in the PDB or in archival technical journals such as Acta Crystallographica F, which are rarely consulted by the biomedical community.

Recognizing the importance of protein structures for the understanding of metabolic diseases in molecular detail and particularly as valuable leads for further functional studies, structure-guided design, and general molecular modeling - JIMD now accepts selected structure reports. The structure determination of human fumarate hydratase (FH) by Picaud and colleagues in this issue provides the inaugural case (Picaud et al. 2011).

B. Rupp $(\bowtie)$

k.k. Hofkristallamt,

Livermore, CA 94551, USA

e-mail: hofkristallamt@gmail.com
FH deficiency is associated with a wide range of metabolic disorders ranging from encephalopathy to renal cancer. More than 107 variants - 93 of them pathogenic - of the enzyme have been described, and based on the crystal structure of the native human enzyme, establishment of structure-function relationships between the various mutants and their clinical phenotypes can be pursued. Structural analysis of the location of the mutations immediately provides some general rationale. The mutations fall into two general clusters; those in the core region primarily affecting overall folding, stability, and integrity of the homotetramer; and those in vicinity of the active site (formed by three different monomer chains) specifically affecting enzyme activity.

Moreover, availability of this high resolution structure now provides guidance for further in silico and in vitro experiments. For single site mutations, the change in binding pocket properties and its functional implications on enzyme activity can often be inferred from in silico modeling. More difficult to predict in silico are effects of mutations on protein-protein interfaces and in allosteric sites - almost always such cases require further experimental structure determination and functional follow-up studies of the mutant. In contrast to the structure-based design of inhibitory drugs, enzyme deficiencies are much harder to address. Even in such cases, the molecular structure may provide avenues for allosteric enhancement of enzyme function.

It is important to realize that not all protein crystal structure models are created equal. While competent peer review generally prevents gross mistakes (Rupp 2006), the suitability of a protein structure for specific modeling and analysis depends on its local features. Even in a high resolution protein structure of good overall quality, some parts (generally surface exposed residues or loops) can be 
poorly defined or plain absent due to flexibility, dynamic movements, or static disorder. Such normally flexible regions can on the other hand be restrained in non-native conformations due to crystal packing contacts - the display of symmetry related neighbors (Hooft et al. 1994) is therefore mandatory when inspecting and analyzing a crystal structure. Similar considerations hold for the establishment of the biologically relevant assembly, which may not be identical to the deposited asymmetric crystallographic unit (Krissinel and Henrick 2007). First means to detect problems in a model region are the analysis of the atomic $B$-factors (a general measure for atomic position displacement - large values imply higher uncertainty) and foremost, inspection of the electron density, which represents the primary crystallographic evidence - the structure is just a model built to fit this electron density. The wwPDB and particularly the Protein Databank in Europe [PDBe, formerly Molecular Structure Database (Velankar et al. 2010)] provide the necessary tools as well as pre-computed electron density (Kleywegt et al. 2004) to examine structures in specific regions of interest.

While the caveats outlined above hint that a complete analysis of protein structure models is not trivial, new tools greatly assist the occasional user to visualize the structure model and to navigate through its relevant parts. For our inaugural example of $\mathrm{FH}$, the authors have provided a webbased, interactive iSee datapack based on the activeICM technology (Raush et al. 2009). Follow the link to the enhanced online version of the manuscript, install the applet, go to Table 1, and click a mutation of interest and the interactive structure viewer maneuvers to the correct site in the model and displays the involved wildtype residues and their interactions. Examine for example the Arg 233 mutations (20-22) in the vicinity of the active site: A substitution of arginine with uncharged residues will interrupt a complex network of hydrogen bonds and charged interactions, which in turn will likely lead to small but significant structural rearrangements affecting the nearby binding pocket - congratulations, you have just accomplished your first structure-function analysis!

Link to interactive FH structure report: http://www. ssiem.org/resources/structures/FH

\section{References}

Berman H, Henrick K, Nakamura H (2003) Announcing the worldwide protein data bank. Nat Struct Biol 10:980

Rupp B (2006) Real-space solution to the problem of full disclosure. Nature 444(7121):817

Hooft RWW, Sander C, Vriend G (1994) Reconstruction of symmetryrelated molecules from protein data bank (PDB) files. J Appl Crystallogr 27(6):1006-1009

Krissinel E, Henrick K (2007) Inference of macromolecular assemblies from crystalline state. J Mol Biol 372:774-797

Velankar S, Best C, Beuth B et al. (2010) PDBe: protein data bank in Europe. Nucleic Acids Res 38:D308-D317

Kleywegt GJ, Harris MR, Zou J-Y et al. (2004) The uppsala electrondensity server. Acta Crystallogr D60:2240-2249

Raush E, Totrov M, Marsden BD et al. (2009) A new method for publishing three-dimensional content. PLoS One 4(10): e7394

Picaud S, Kavanagh KL, Yue W, Gileadi O, Sacchettini J, Oppermann U (2011) Structural basis of fumarate hydratase deficiency. J Inherit Metab Dis. doi:10.1007/s10545-011-9294-8 